\title{
Assessment of a silicon detector for pulsed neutron scattering experiments
}

\author{
M. Tardocchi ${ }^{\mathrm{a}, *}$, C. Arnaboldi ${ }^{\mathrm{b}}$, G. Gorini ${ }^{\mathrm{a}}, \mathrm{S}$. Imberti ${ }^{\mathrm{a}}, \mathrm{G}$. Pessina $^{\mathrm{b}}$, \\ E. Previtali ${ }^{\mathrm{b}}$, C. Andreani ${ }^{\mathrm{c}}$, A. Pietropaolo ${ }^{\mathrm{c}}$, R. Senesi ${ }^{\mathrm{c}}$ \\ a INFM, UdR Milano-Bicocca and Dipartimento di Fisica "G. Occhialini”, Università degli Studi di Milano-Bicocca, Milano, Italy \\ ${ }^{\mathrm{b}}$ INFN, Sezione di Milano and Dipartimento di Fisica "G. Occhialini”, Università degli Studi di Milano-Bicocca, Milano, Italy \\ ${ }^{\mathrm{c}}$ Università degli Studi di Roma Tor Vergata, Dipartimento di Fisica and INFM, UdR Roma Tor Vergata, Rome, Italy
}

\begin{abstract}
Resonance detectors (RD) are being developed for neutron spectroscopy in the epithermal energy region at spallation neutron sources. Different choices of converter foils and gamma spectrometers are being compared as part of an optimization and selection process within the TECHNI project. This paper reports on the design of a silicon detector system and some preliminary tests on the VESUVIO spectrometer. The detector has a good efficiency in the X-ray energy range, where two intense photon peaks (at 12 and $48 \mathrm{keV}$ ) are expected to be emitted following neutron capture in a uranium converter foil. The detector energy resolution has been improved by nitrogen vapor cooling of the silicon chip and by careful design of the preamplifier electronics. Neutron time of flight spectra have been measured on VESUVIO when the converter foil is placed in the neutron beam. In that case, the detector response is dominated by a continuum due to Compton detection of gammas of higher energy. These results provide a basis for a critical assessment of the applicability of silicon detectors for RD measurements of epithermal neutrons.
\end{abstract}

(C) 2004 Elsevier B.V. All rights reserved.

PACS: $29.30 . \mathrm{Hs} ; 29.30 . \mathrm{Kv} ; 29.40 . \mathrm{Wk} ; 61.12 . \mathrm{Ex}$

Keywords: Neutron spectroscopy; Epithermal neutrons; Resonance detector; Silicon detector

\section{Introduction}

Resonance detectors (RD) [1] are being developed for epithermal neutron spectroscopy at pulsed neutron sources. The development of new instrumentation for neutron detection is motivated by the interest in extending the (q, $\omega$ ) kinematical region that can be accessed in today's neutron

\footnotetext{
*Corresponding author. Tel.: + 39-02-6448-2326; fax: + 3902-6448-2367.

Email-address: marco.tardocchi@mib.infn.it, marco.tardocchi@mi.infn.it (M. Tardocchi).
}

scattering experiments [2]. In particular, to access the kinematical region of both high and low momentum transfer coupled to high-energy transfer one requires detection of neutrons of energy in the range $1-100 \mathrm{eV}$ [3]. The $\mathrm{RD}$ configuration on a pulse neutron source represents the most effective method to perform experiments at such high neutron energies.

In the RD configuration neutron detection is performed in a two-step process: (i) neutron resonance absorption in a converter foil (e.g. ${ }^{238} \mathrm{U}$ ) which absorb neutrons of energies defined by the nuclear resonances of the converter (ii) 


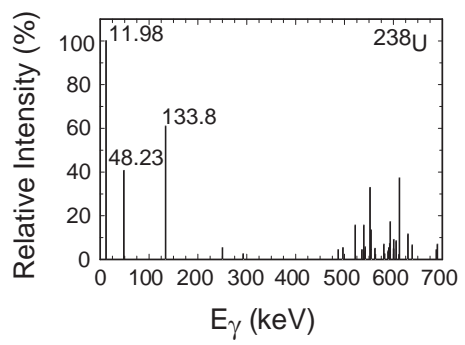

Fig. 1. Relative intensities in the energy range below $500 \mathrm{keV}$ of the radiative capture $\gamma$ 's following thermal neutron capture on ${ }^{238} \mathrm{U}$. The energies of the four highest intensity resonances are indicated.

detection of the prompt radiative capture $\gamma$ - and $\mathrm{X}$-ray radiation. The photon detector by detecting the prompt photons records the arrival time of the neutron absorbed in the converter foil. The time of flight technique, available at a pulse source, allows thus to reconstruct the whole kinematics of the scattering process. The choice of the photon detector depends on the $\gamma$ emission yield of the converter foil in the energy range where the detector shows good efficiency. In the case of ${ }^{238} \mathrm{U}$, the literature available for thermal neutron absorption indicates that the most intense emitted $\gamma$ line is expected at $12 \mathrm{keV}$, as shown in Fig. 1 . Among the several neutron converters and photons detectors tested, reported in Refs. [3-6], we have designed a silicon detector system and tested it in preliminary measurements on the neutron spectrometer VESUVIO [7], installed at the ISIS pulsed neutron source. The response of a cooled Si-diode to neutron capture emission from ${ }^{238} \mathrm{U}$ in the energy range $1-100 \mathrm{eV}$ has been first characterized by measuring neutron time of flight spectra with the ${ }^{238} \mathrm{U}$ foil placed at the sample position. Second, the performance of the Si-diode combined to a ${ }^{238} \mathrm{U}$ foil in the RD configuration has been assessed by measuring neutrons scattered from a lead sample.

\section{Experimental set-up}

The experiment was performed on the inverse geometry neutron spectrometer VESUVIO, in the RD configuration (Fig. 2). The Si photo-diode

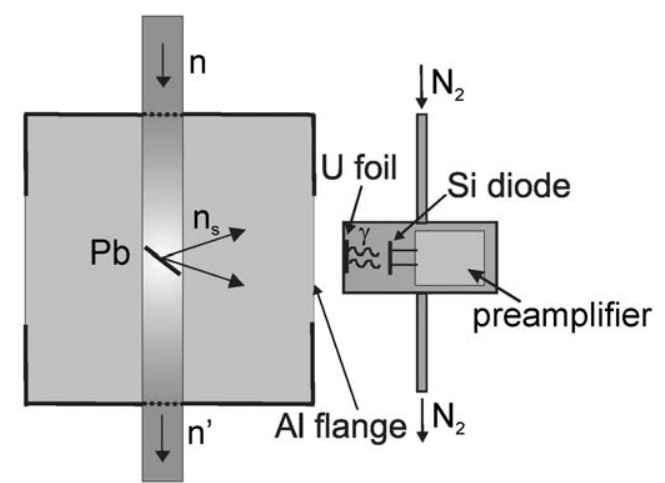

Fig. 2. Schematics view of Vesuvio in the RD configuration. The neutrons $(\mathrm{n})$ incoming at the scattering sample $(\mathrm{Pb})$ are scattered $\left(n_{s}\right)$ and detected by the combination of a ${ }^{238} \mathrm{U}$ foil and a Si diode.

$\left(18 \times 18 \times 0.5 \mathrm{~mm}^{3}\right)$ was located with the ${ }^{238} \mathrm{U}$ foil $\left(30 \times 30 \times 0.075 \mathrm{~mm}^{3}\right)$ and the preamplifier electronics in an light tight aluminum box. The box served also for the flow of nitrogen $\left(\mathrm{N}_{2}\right)$ vapor which was used to cool down the detector to about $-30^{\circ} \mathrm{C}$. The cooling was controlled by varying the current running through a resistor inserted in a $\mathrm{N}_{2}$ dewar, located at about $5 \mathrm{~m}$ outside the experimental hall. The mechanism proved to be effective although it required constant human supervision to obtain an acceptable temperature stabilization. Some auxiliary electronics such as $\mathrm{HV}$ for the Sidiode, $\pm 6 \mathrm{~V}$ for the preamplifier and the cooling control were located outside the experimental hall.

A dedicated preamplifier was designed for the Si-diode. The preamplifier had at its input a low noise JFET transistor with an input capacitance of about $60 \mathrm{pF}$. A dominant pole configuration was adopted with a feedback capacitance of $1 \mathrm{pF}$ shunted with $10 \mathrm{G} \Omega$ resistor. The current noise was kept so small in order to allow for the adoption of a large shaping time. Because of the expected small amplitude of the detector signals, a second stage preamplifier with a large voltage gain, $500 \mathrm{~V} / \mathrm{V}$, was also developed to feed the shaper circuit located in the acquisition room. The final shaping time constant adopted was about $5 \mu \mathrm{s}$, which represented a compromise between noise and acquisition rate. Cooling from ambient temperature to $-30^{\circ} \mathrm{C}$ allowed to reduce the 
detector leakage current from $10 \mathrm{nA}$ to $150 \mathrm{pA}$ and, consequently, the overall energy resolution was improved from 8 to $4 \mathrm{keV}$ (FWHM). The formed analog pulse from the preamplifier was discriminated and the logic signal was sent to the ISIS data acquisition which, by recording the arrival time of each signal, produced the neutron time of flight histogram.

\section{Results and discussion}

Preliminary to the measurement, energy calibrations have been performed with a ${ }^{57}$ Co radioactive source which was positioned inside the detector box; the source provided two identifiable $\gamma$ lines at 122 and $136 \mathrm{keV}$. An example of pulse height spectrum from the Si-diode is shown in Fig. 3. The energy resolution of the line at $122 \mathrm{keV}$ is about $4 \mathrm{keV}$ (FWHM). We observe that there is a significant level of background at energies below $100 \mathrm{keV}$ which is due to the natural radioactivity of the ${ }^{238} \mathrm{U}$ foil as well as to the impurities present in the ${ }^{57} \mathrm{Co}$ source.

The response of the Si-diode to the neutron capture emission from ${ }^{238} \mathrm{U}$ is shown in the neutron time of flight spectrum of Fig. 4a, collected with the ${ }^{238} \mathrm{U}$ converter located at the sample position. Four ${ }^{238} \mathrm{U}$ resonances can be distinguished, up to neutron energies of $66 \mathrm{eV}$. The detector pulse-height response is dominated by a rather flat continuum due to Compton detection of $\gamma$ 's of energy above $60 \mathrm{keV}$; at such energies Compton interaction in the Si-diode is more

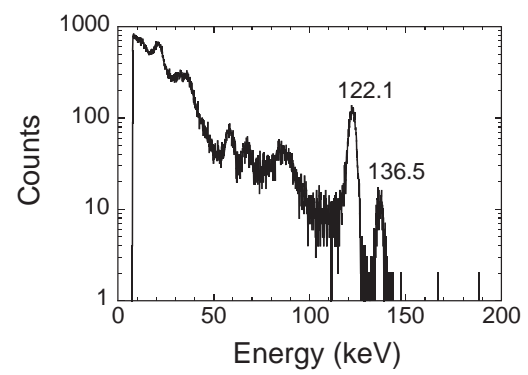

Fig. 3. Calibration spectrum of the Si-diode obtained with a ${ }^{57} \mathrm{Co}$ source located inside the detector box.

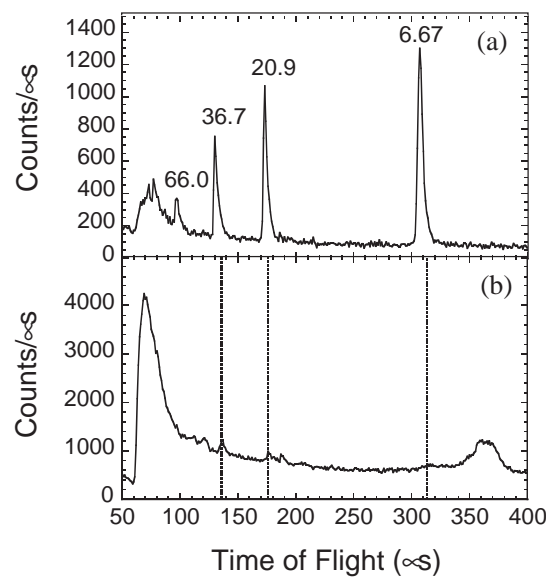

Fig. 4. Time of flight spectra recorded with: (a) ${ }^{238} \mathrm{U}$ foil placed at the sample position; the four labeled peaks correspond to ${ }^{238} \mathrm{U}$ resonances. (b) Polycrystalline lead sample; the dashed lines indicate the expected positions of the first ${ }^{238} \mathrm{U}$ resonances.

probable than photoelectric absorption. In comparison, measurements in the RD configuration from a polycrystalline lead sample (Fig. 4b) have shown that the background (neutron and $\gamma$ ) prevents a clear detection of the ${ }^{238} \mathrm{U}$ signal peaks. A broad structure is present at about $t=360 \mu \mathrm{s}$ which can be identified as a ${ }^{197} \mathrm{Au}$ resonance located at $E_{\mathrm{n}}=4.9 \mathrm{eV}$. Gold is used in the Si-diode for instance in the cathode metallization, lead pins, etc.

The obtained results seem to indicate that, despite signals have been observed in the measurement with the ${ }^{238} \mathrm{U}$ foil at the sample position, the very low $\gamma$ energy region $\left(E_{\gamma}<50 \mathrm{keV}\right)$ is not favorable for the development of epithermal neutron detectors in the RD configuration. Indeed measurements at higher $\gamma$ energy regions $\left(E_{\gamma}>500 \mathrm{keV}\right)$ have shown a much better signal to background ratio [3]. To further exploit the very low $\gamma$-energy region one would need to reduce the background, for instance using a different detection principle.

\section{Acknowledgements}

Work performed with financial support by the European Community-Access to Research Infrastructure action of the Improving Human Potential Programme. 


\section{References}

[1] D.R. Allen, et al., J. Phys. E: Sci. Instr. 13 (1980) 639.

[2] R.J. Newport, et al., Nucl. Instrum. and Meth. 224 (1984) 120.

[3] A. Pietropaolo, et al., Epithermal neutron scattering at high- $w$ and low- $q$ on VESUVIO in the resonance detector spectrometer configuration: solid state detectors and scintillators, Physica B, accepted.
[4] C. Andreani, et al., Nucl. Instrum. and Meth. A 481 (2002) 509.

[5] C. Andreani, et al., Appl. Phys. A 78 (2004) 903.

[6] M. Tardocchi, et al., Cadmium-zinc-telluride photon detector for epithermal neutron spectroscopy - pulse height response characterisation, Nucl. Instrum. and Meth. A, accepted.

[7] R. Senesi, et al., Physica B 276-278 (2000) 200. 